\title{
Percutaneous transhepatic dual chamber pacing in children with Fontan circulation
}

\author{
S S Adwani, N Sreeram, J V DeGiovanni
}

\begin{abstract}
Permanent pacing is often required following the Fontan operation and is usually performed epicardially as there is no direct access to the ventricle from the systemic veins. Dual chamber endocardial pacing was achieved by the transhepatic approach in two children with Fontan circulation. The patients were a 7 year old boy with left atrial isomerism, single ventricle with pulmonary stenosis, interrupted inferior vena caval vein with azygous continuation, and direct drainage of the hepatic veins to the right sided atrium, and a 6 year old girl with tricuspid atresia. This approach to endocardial pacemaker implantation is potentially of considerable value in patients who do not have direct access to the ventricle from the systemic veins.
\end{abstract}

(Heart 1997;77:574-575)

Keywords: transhepatic endocardial pacing; children; Fontan circulation

Permanent pacing following the Fontan operation for single ventricle physiology is usually performed epicardially ${ }^{1}$ as there is no direct access to the ventricle from the systemic veins. Other approaches described have been pacing via the coronary sinus ${ }^{2}$ and extraperitoneal approach to the inferior vena caval vein by abdominal surgery. ${ }^{3}$ We describe two patients with Fontan circulation who underwent percutaneous dual chamber pacemaker implantation using the transhepatic route.

\section{Case 1}

A 7 year old boy with left atrial isomerism, single ventricle with pulmonary stenosis, interrupted inferior vena caval vein with azygous continuation, and direct drainage of the hepatic veins to the right sided atrium developed complete heart block five years after palliative superior cavopulmonary shunt. $\mathrm{He}$ had decreased exercise tolerance and exercise induced ventricular tachycardia for which it was decided to insert a dual chamber endocardial pacemaker via the transhepatic route. Using ultrasound guidance the hepatic vein was punctured twice and two sheaths $(8 \mathrm{~F}$ and $7 \mathrm{~F}$ ) were positioned in the right sided atrium. A 7 F Capsure fined steroid eluting lead
(Medtronic 4023, Minneapolis, USA) was inserted in the ventricular apex and a $6 \mathrm{~F}$ screw-in unipolar lead (Osypka KY 1167C, Grenzach-Wyhlen, Germany) was secured in the atrium. The pacemaker generator was buried in the anterior abdominal wall anterior to the rectus abdominis muscle behind the rectus sheath. Ultrasound of the liver showed no evidence of liver laceration or haemorrhage. Follow up three months later showed satisfactory pacing and sensing with no evidence of lead displacement.

\section{Case 2}

A 6 year old girl with tricuspid atresia developed complete heart block following the Fontan operation (atriopulmonary connection using a Goretex intra-atrial baffle). Two years later she developed increasing fatigue with decreased effort tolerance that was attributed to chronotrope incompetence. It was decided to implant a dual chamber endocardial pacemaker using the transhepatic route. Under ultrasound guidance the hepatic vein was cannulated and an $8 \mathrm{~F}$ Mullins transseptal sheath was positioned in the right atrium. Using transoesophageal echocardiographic guidance, the Goretex baffle was punctured using a transseptal needle and a stiff guidewire was inserted in the left ventricle. This was then exchanged for an $8 \mathrm{~F}$ "peel away" sheath through which a $7 \mathrm{~F}$ Capsure fined steroid eluting lead (Medtronic 4023) was introduced and positioned in the left ventricle. Through the same sheath, a second wire was introduced into the right atrium, followed by introduction of a $9 \mathrm{~F}$ sheath. A $9 \mathrm{~F}$ screw-in atrial J lead (Medtronic 4568) was secured in the right atrium. The generator was buried in the anterior abdominal wall anterior to the rectus abdominis muscle behind the rectus sheath. Routine anticoagulation with warfarin was recommenced the following day. The patient developed a subcutaneous haematoma in the anterior abdominal wall 48 hours later which settled spontaneously. Computed tomography showed no evidence of liver laceration or bleed in the pacemaker pocket. Outpatient review one month later showed satisfactory pacing and sensing with no evidence of lead displacement.

\section{Discussion}

Arrhythmias are common following the Fontan operation, and some patients will eventually require permanent pacemaker
J V DeGiovanni Dr DeGiovanni.

Accepted for publication 3 March 1997 


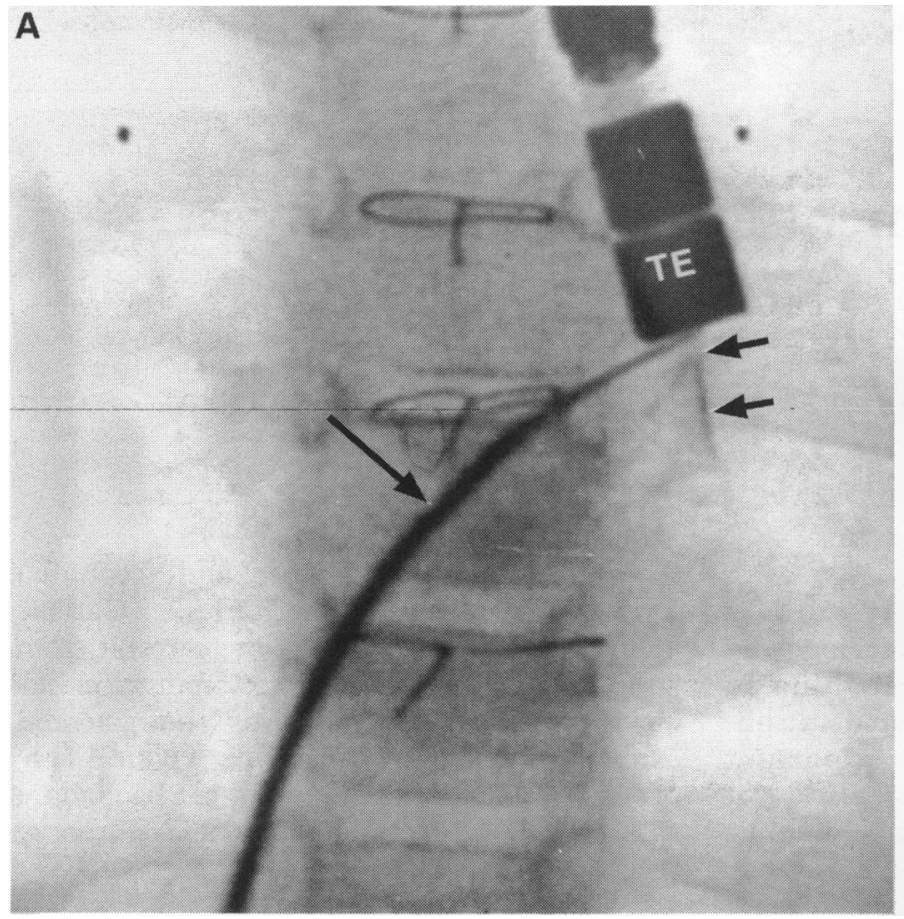

(A) Angiography showing perpendicular relation of the transseptal needle (large arrow) to the Goretex baffle (small arrows) via the transhepatic route. The atrial septum has been tagged with a small amount of contrast. TE, tip of transesophageal ultrasound probe. (B) $x$-ray showing atrial and ventricular location of the pacing wires with a generous intracardiac loop and generator in the anterior abdominal wall.

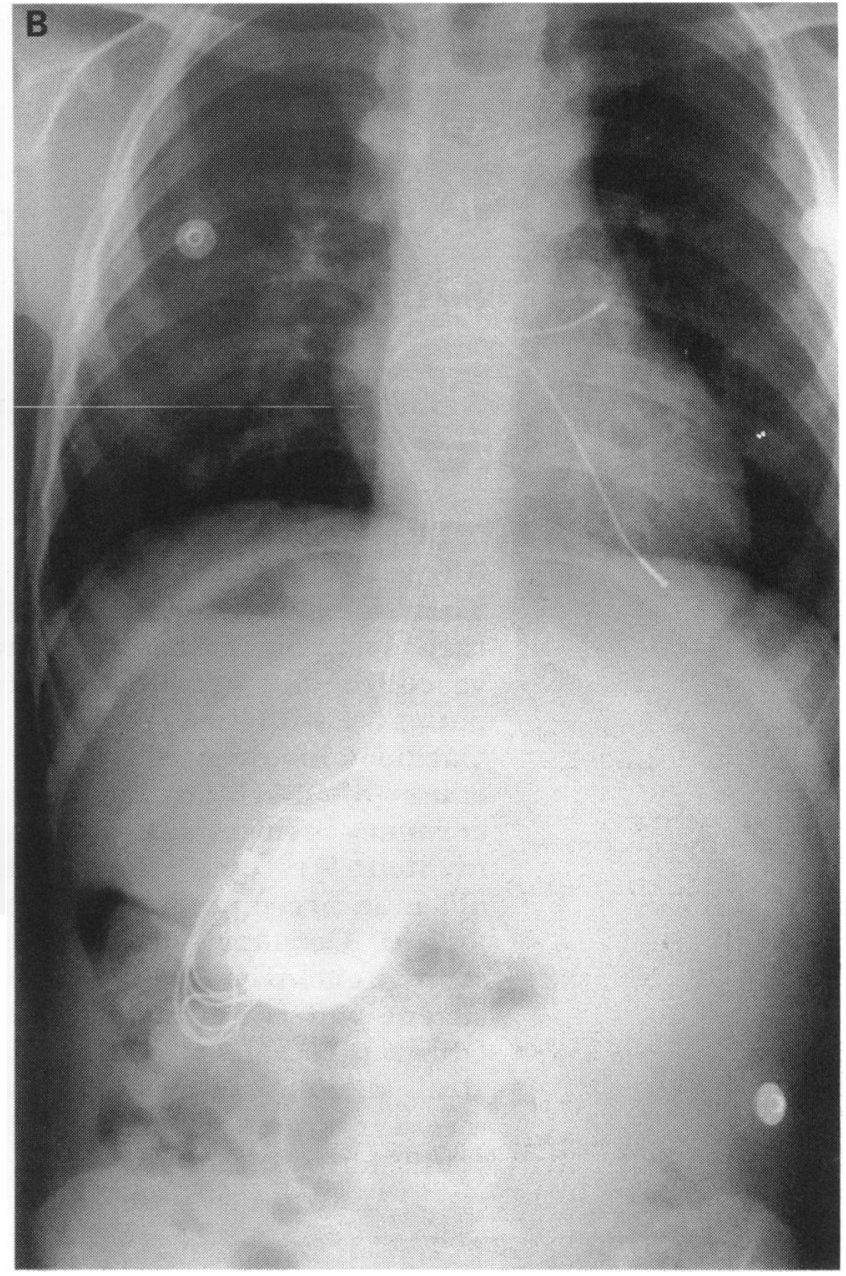

implantation. The preferred route is epicardial pacing, due to the difficulties of transvenous access to the single ventricle from the systemic venous side. Epicardial pacing, however, requires a thoracotomy and is sometimes associated with high pacing thresholds and sensing problems. Percutaneous ventricular pacing is sometimes possible via the coronary sinus, ${ }^{2}$ but the coronary sinus is often left to drain into the pulmonary venous atrium, making it inaccessible. Additionally, there is a risk of coronary sinus thrombosis, and future lead revisions can be technically very complicated.

The transhepatic approach has been used to obtain long term central venous access and for cardiac catheterisation in children and adults ${ }^{4}$ in whom standard percutaneous venous access sites may not be available. Recently, this approach has been described for endocardial atrial pacing in a 4 year old male following Senning procedure for transposition of the great arteries where conventional venous access was not possible due to multiple venous occlusions. ${ }^{5}$ As the distance to the atrial septum is short, the transhepatic approach allows "peel away" sheaths of standard lengths to be used to introduce the pacemaker wires. An added advantage with transseptal puncture is that the course of the transseptal needle from the middle hepatic vein is almost directly perpendicular to the atrial septum. This allowed continuous contact between the needle and the atrial baffle (figure), so that constant pressure could be applied to the needle to enable perforation of the Goretex baffle.
Lead revision following transhepatic pacemaker implants should be possible as the hepatic vein can be punctured again. Also, as the path to the atrium and ventricle is short, a generous loop of the atrial and ventricular lead can be left allowing for the patient's growth and less frequent need for revision (fig). As there is possibility of systemic thromboembolism from a lead in the systemic ventricle, ${ }^{6}$ anticoagulation should be seriously considered. Bleeding into the abdominal wall in the second patient most likely resulted from traumatic perforation of a blood vessel during percutaneous entry. There is, however, a theoretical risk of bleeding into the liver capsule following the transhepatic approach, although this did not occur in either patient.

In conclusion, successful percutaneous dual chamber endocardial pacing can be achieved in the Fontan circulation using the transhepatic approach.

1 Fishberger SB, Wernovsky G, Gentles TL, Gamble WJ, Gauvreau K, Burnett J, et al. Long-term outcome in patients with pacemakers following the Fontan operation. Am f Cardiol 1996;77:887-9.

2 Blackburn MEC, Gibbs JL. Ventricular pacing from the coronary sinus of a patient with a Fontan circulation. $\mathrm{Br}$ Heart $\mathcal{F}$ 1993;70:578-9.

3 West JN, Shearmann CP, Gammage M. Permanent pacemaker positioning via the IVC in a case single ventricle with loss of RA vena cava continuity. PACE 1993;16: 1753-5.

4 Shim D, Lloyd TR, Cho KJ, Moorehead CP, BeekmanIII RH. Transhepatic cardiac catheterization in children. Circulation 1995;92:1526-30.

5 Fishberger SB, Camunas J, Rodriguez-Femandez $H$, Sommer RJ. Permanent pacemaker lead implantation via the transhepatic route. PACE 1996;19:1124-5.

6 Sharifi M, Sorkin R, Sharifi V, Lakier JB. Inadvertent malposition of a transvenous-inserted pacing lead in the left ventricular chamber. Am ₹ Cardiol 1995;76:92-5. 